\title{
Evaluation of Tip Surface Collision Count as a Measure of Fibreoptic Scope Handling Skills: A Randomized, Cross-Over Manikin Study
}

\author{
Mari H. Roberts, ${ }^{1}$ Elizabeth K. Boucher, ${ }^{1}$ Michael Lim, ${ }^{1}$ \\ Antony R. Wilkes, ${ }^{2}$ and Iljaz Hodzovic ${ }^{2}$ \\ ${ }^{1}$ Department of Anaesthetics and Intensive Care Medicine, University Hospital of Wales, Cardiff CF14 4XN, UK \\ ${ }^{2}$ Department of Anaesthetics, Intensive Care and Pain Medicine, School of Medicine, Cardiff University, Cardiff CF14 4XN, UK
}

Correspondence should be addressed to Iljaz Hodzovic; hodzovic@cardiff.ac.uk

Received 6 August 2014; Revised 12 September 2014; Accepted 14 September 2014; Published 2 October 2014

Academic Editor: Thomas Engelhardt

Copyright (C) 2014 Mari H. Roberts et al. This is an open access article distributed under the Creative Commons Attribution License, which permits unrestricted use, distribution, and reproduction in any medium, provided the original work is properly cited.

Background. In this pilot study, we evaluated tip collisions against three commonly used measures of fibreoptic scope handling skills. Methods. Seventy-seven anaesthetists were recruited to perform a standardized task on an Oxford Box and a modified AirSim manikin. Collision count was correlated against time to scope placement, a global rating scale score, and up-to-date fibreoptic experience. Results. Strong and moderate correlations were found between collision count and task completion time for the Oxford Box $(\rho=0.730, P<0.0001)$ and AirSim manikin $(\rho=0.405, P<0.0001)$, respectively. Moderate correlation was found between collision count and global rating scale score for the Oxford Box $(\rho=-0.545, P<0.0001)$ and AirSim manikin $(\rho=-0.500, P<$ 0.0001). Mild and moderate correlations were found between collision count and fibreoptic experience on the Oxford Box ( $\rho=$ $-0.240, P=0.041)$ and AirSim manikin $(\rho=-0.423, P<0.0001)$, respectively. Conclusions. This study infers that collision count may be used as a measure of fibreoptic scope handling skills in simulation training. Using this outcome in addition to other measures of performance may improve accuracy and precision of fibreoptic scope placement.

\section{Introduction}

Fibreoptic intubation is a core anaesthetic airway skill. It is internationally accepted as an important tool in the management of the difficult airway $[1,2]$. Traditionally it has been taught using the apprenticeship model. However, anaesthetists may require 25 fibreoptic training opportunities to gain proficiency [3] when the number of patients with anticipated difficult laryngoscopy requiring fibreoptic intubation is limited. Trainees find it difficult to gain sufficient patient experience; consequently the skill is increasingly taught on manikins and other teaching devices.

Previous studies on fibreoptic intubation training have used outcome measures such as success rates $[3,4]$ and/or time to fibreoptic scope guided tracheal intubation [4-6]. However, these quantitative measures give limited information on the subject's performance and skill [6]. Other outcome measures, such as global assessment scales and check lists $[3,4]$, have been used to evaluate fibreoptic scope intubation training.

Maintaining a clear view and avoiding collision with pathological structures may be more important than speed of intubation and may be a better measure of fibreoptic scope handling skill [7]. The number of times the bronchoscope makes contact with the airway wall with loss of view has been used as an outcome measure of handling skills in both patient $[8]$ and simulation $[9,10]$ studies. However, we could find no publication evaluating the usefulness of counting collisions as a measure of handling skills.

Male gender, right hand dominance, and computer game experience have been shown to be associated with superior laparoscopic performance amongst surgeons [11]. We could not find similar data related to fibreoptic scope handling skills amongst anaesthetists. 


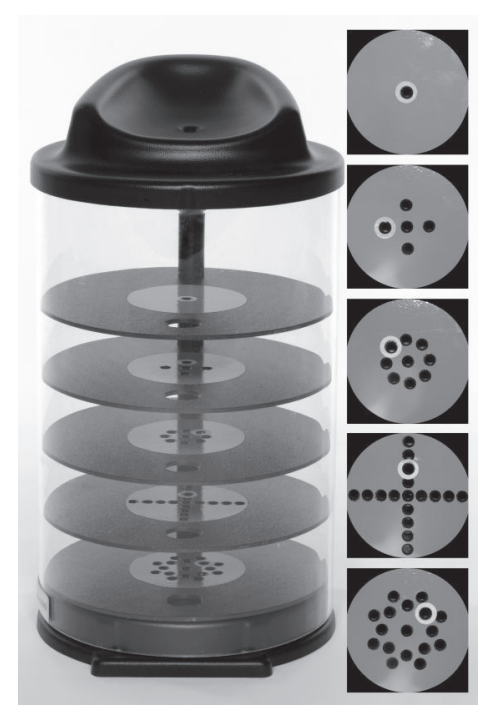

Figure 1: Oxford Fibreoptic Training Box (inserts show fibreoptic views).

We hypothesised that collision count (the number of times the view was lost for any length of time) can be used as a measure of fibreoptic handling skill. Our aim was to evaluate the collision count against three commonly used outcome measures: time to fibreoptic scope placement, a global rating scale, and previous fibreoptic experience. We also assessed the effect of gender, hand dominance, and computer game experience on the tip collision count and fibreoptic placement time.

\section{Methods}

Enquiries were made to the South East Wales Research Ethics Committee regarding the necessity for ethics committee approval, and on the 26th August 2009, it was decided by the chairman of the South East Wales Research Ethics Committee Dr. D. E. B. Powell that "the project should be regarded as a "service development" and therefore does not require ethical review by an NHS Research Ethics Committee."

Written informed consent was obtained from 77 anaesthetists working in the South Wales region (University Hospital of Wales and Royal Gwent Hospital). We included volunteers with a wide range of fibreoptic experience, from those with no experience to those who use a fibreoptic scope on a daily basis.

Volunteer's gender, grade, years of experience in anaesthesia, and up-to-date experience with fibreoptic intubation on manikins and patients (awake and anaesthetised) were noted. Data on hand dominance and preferred hand for controlling the fibreoptic scope (controlling hand) was collected retrospectively. Experience with computer games, defined as playing games requiring hand eye coordination on a regular basis, was also recorded.

The Oxford Fibreoptic Training Box (Pharmabotics Ltd., Winchester, UK) (Figure 1) is a portable model used for fibreoptic scope manipulation training. The Oxford Box uses

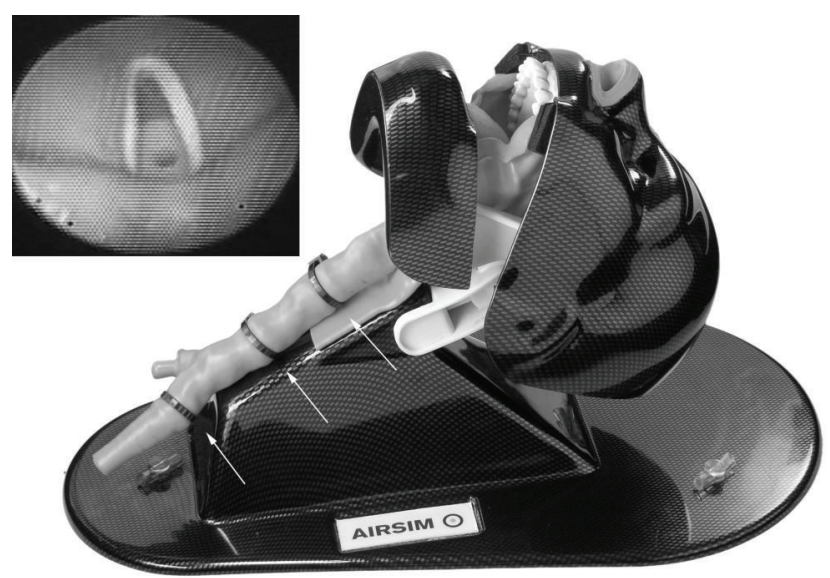

FIgURE 2: AirSim manikin with modified airway (indicated by arrows). Insert shows fibreoptic scope view.

a choose-the-hole training method designed to enhance fibreoptic scope manipulation skills. This training method enhances directional handling of the fibreoptic scope and has been shown to be an effective teaching tool in fibreoptic intubation [4].

The AirSim Multi manikin (Trucorp Ltd., Belfast, Northern Ireland) (Figure 2) is an anatomically realistic model with visually accurate internal features, including a nasal passage with turbinates. The manikin is moulded in one piece and is constructed from a material that delivers good tactile feedback throughout the nasal passage and airway.

The volunteers were given time to familiarise themselves with the fibreoptic scope (Pentax FI-10RBS, Pentax House, Slough SL3 8PN, UK) but no preliminary runs were allowed. Each volunteer was asked to complete two standardised tasks-one on the Oxford Box and one on a modified AirSim manikin, in a computer generated randomized order (Modified Excel Programme). The task on the Oxford Box involved manipulating the fibreoptic scope through labelled holes at five levels (Figure 1). Task completion time was measured from the time the tip of the fibreoptic scope passed through the first hole until the tip emerged through the marked hole on the fifth level (completion time: box). The task on the AirSim manikin involved manipulating the fibreoptic scope, via the nasal route, into the trachea and left main bronchus. The manikin was modified in order to increase the complexity of the task. This was done by fixing plastic "washers" (internal diameter $5 \mathrm{~mm}$ ) with tapered edges along the bronchial tree in three places: below the vocal cords, above the carina, and in the left main bronchus (Figure 2). The task completion time for the AirSim manikin was recorded from the time the fibreoptic scope entered the nose to the time it appeared through the open end of the left main bronchus (completion time: manikin). A resting period of 30 seconds was given to each volunteer between the two tasks.

The handling of the fibreoptic scope during both tasks was recorded by the researchers on a Five-Point Global Rating Scale of Fibreoptic Bronchoscope Manipulation Ability 
TABLE 1: Distribution of gender, hand dominance, fibreoptic scope controlling hand, and playing of computer games in 72 volunteers.

\begin{tabular}{lc}
\hline $\begin{array}{l}\text { Hand dominance } \\
\text { Right : left }\end{array}$ & $66: 6$ \\
\hline $\begin{array}{l}\text { Controlling hand } \\
\text { Dominant: nondominant }\end{array}$ & $48: 24$ \\
\hline $\begin{array}{l}\text { Playing computer games } \\
\text { Yes : no }\end{array}$ & $15: 57$ \\
\hline
\end{tabular}

(Global Rating Scale) previously used by other investigators $[4,12]$ (Table 3 ). The number of tip collisions was independently counted from a video recording of the fibreoptic view by two researchers, who were blinded to the volunteer's identity. Each researcher counted the number of collisions three times, for each of the two tasks. The average of the six counts was calculated (collision count). A tip surface collision was defined as complete or partial loss of view for any length of time. Volunteers who did not complete the task were excluded from the final analysis.

Our primary aim was to correlate the completion time with the collision count obtained during successful completion of both the Oxford Box and modified AirSim manikin tasks. Our secondary aims were to correlate the collision count with the global rating scale score and with previous fibreoptic intubation experience. In addition, we assessed the effect of gender, hand dominance, and computer game experience on the completion time and collision count of the two tasks.

Statistical Analysis. The Spearman rank order correlation test was used to test for significant correlations. Mann-Whitney $U$ test was used to analyse continuous data that was not normally distributed. A $P$ value of $<0.05$ was considered significant. SPSS v 16 (SPSS Inc., Chicago, IL, 60606) and Minitab v 16 (Minitab Inc, State College, PA 16801-3008) were used to analyze the data.

\section{Results}

Thirty-five consultants and 42 trainees participated in the study (male: female $=46: 31$ ). The median (IQR [range]) number of years of anaesthetic experience of the volunteers was $8(3-15[0.5-38])$. Four volunteers did not complete the Oxford Box task and one did not complete the AirSim manikin task. All uncompleted attempts were stopped at the volunteers' request due to their perceived inability to complete the task. Their results were not included in the correlation calculations.

Data on distribution of hand dominance, controlling hand, and computer game experience is presented in Table 1. The data for the above three variables was collected retrospectively. Five of the 77 volunteers did not respond.

Volunteers' fibreoptic experience ((median (IQR [range])) number of fibreoptic scope uses) on manikins, anaesthetised, and awake patients was 7 (2-20 [0-100]), 20 $(5-30[0-200])$, and $10(2-22[0-100])$, respectively. There
TABLE 2: Spearman Rank Correlations, $\rho$, of collision count for the Oxford Box and AirSim manikin tasks.

\begin{tabular}{lcccc}
\hline & \multicolumn{2}{c}{ Oxford box } & \multicolumn{2}{c}{ AirSim manikin } \\
& $\rho$ & $P$ value & $\rho$ & $P$ value \\
\hline GRS & -0.545 & $<0.0001$ & -0.500 & $<0.0001$ \\
FO experience & -0.240 & $=0.041$ & -0.423 & $<0.0001$ \\
\hline
\end{tabular}

GRS $=$ global rating scale, FO experience $=$ fibreoptic experience.

TABLE 3: Five-point global rating scale of fibreoptic bronchoscope manipulation ability.

\begin{tabular}{lcl}
\hline \multicolumn{1}{c}{1} & \multicolumn{1}{c}{3} & \multicolumn{1}{c}{5} \\
\hline $\begin{array}{l}\text { Repeatedly makes } \\
\text { tentative or awkward } \\
\text { moves with }\end{array}$ & $\begin{array}{l}\text { Competent use of } \\
\text { instruments but } \\
\text { bronchoscope by } \\
\begin{array}{l}\text { occasionally appears } \\
\text { scope }\end{array}\end{array}$ & $\begin{array}{l}\text { Fluid } \\
\text { manipulation of } \\
\text { bronchoscope } \\
\text { and no }\end{array}$ \\
very poor & Competent & $\begin{array}{l}\text { awkwardness } \\
\text { Clearly superior }\end{array}$ \\
\hline
\end{tabular}

was no significant difference between males (50 (20-90 [0$303]))$ and females $(36(18-55[0-230]))$ in total (manikins and patients) fibreoptic experience $(P=0.19)$. Seventy-four of the 77 participants $(96 \%)$ had used the fibreoptic scope in the preceding six months and 37/77 (48\%) had used it in the preceding month.

Task completion times (median (IQR [range])) for the Oxford Box and AirSim manikin were 119 (83-204 [35-451]) and 85 (67-102 [35-266]) seconds, respectively. Collision count (median (IQR [range])) for the Oxford Box was 8 (5-15 [1-40]) and for the AirSim manikin 4 (2-5 [0-11]).

The Spearman rank correlation between collision count and completion time for the Oxford Box was $\rho=0.730(P<$ $0.0001)$ and AirSim manikin $\rho=0.405(P<0.0001)$. Visual illustrations of these correlations for the Oxford Box and manikin are shown in Figures 3 and 4, respectively. Correlation between the Oxford Box and manikin completion times was $\rho=0.4(P<0.01)$ and collision counts were $\rho=0.5(P<0.01)$.

Correlation between global rating scale score and previous fibreoptic scope experience, for each task, is shown in Table 2.

Males completed the Oxford Box task significantly faster than females. The median (IQR [range]) completion times for males and females were $100 \mathrm{~s}(69-189$ [35-451]) and $175 \mathrm{~s}$ (107-235 [75-451]), respectively $(P=0.002)$. There was no significant difference between the two genders in the time taken to complete the AirSim manikin task $(P=0.057)$ or in the collision count for the Oxford Box $(P=0.139)$ or the manikin $(P=0.832)$. The difference between left- and righthanded volunteers in completion time and collision count was not significant for either task.

Regular playing of computer games did not have a significant effect on the Oxford Box completion time $(P=0.147)$ or collision count $(P=0.219)$. Similarly, performance on the AirSim manikin was not significantly different between the two groups with regard to completion time $(P=0.241)$ or 


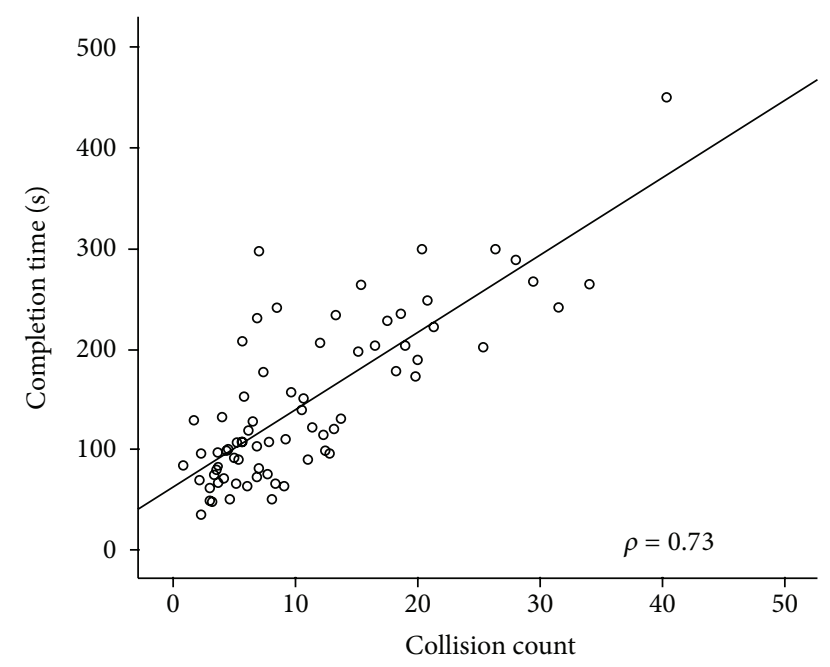

FIGURE 3: Correlation of collision count with completion time for the Oxford Box.

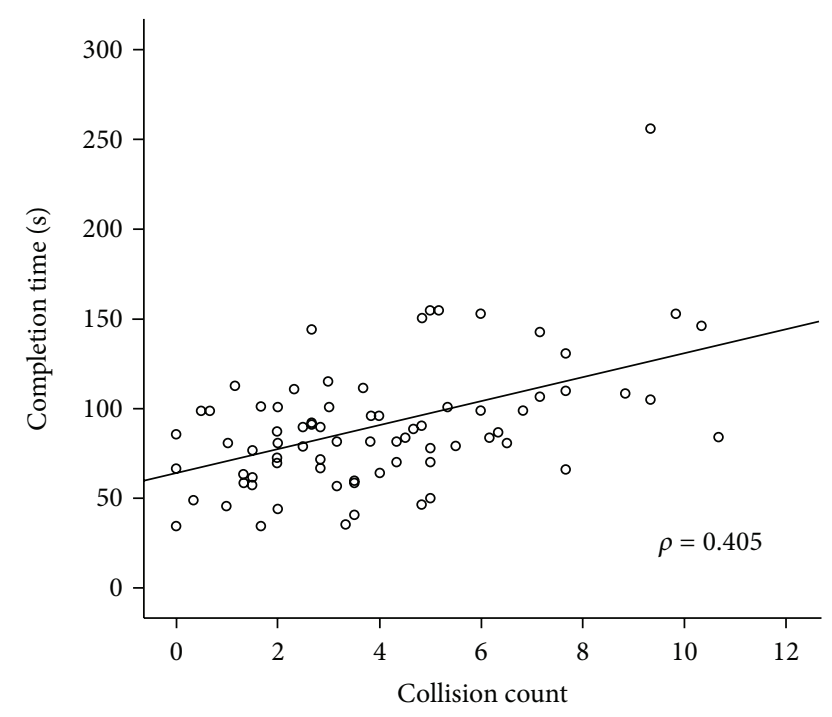

FIgURE 4: Correlation of collision count with completion time for the AirSim manikin.

collision count $(P=0.501)$. However, within a subgroup of 12 novices (less than 15 fibreoptic intubations, manikins and patients) there was a significant difference in the completion time on the Oxford Box between the six that played regular computer games and the six that did not; median (IQR [range]) times were $77 \mathrm{~s}(72-90$ [65-173]) and $220 \mathrm{~s}$ (157265 [91-297]), respectively $(P=0.009)$. No significant difference was found within the novice subgroup for the AirSim manikin completion time $(P=0.699)$.

\section{Discussion}

This study demonstrated strong and moderate correlations between collision count and task completion time on the Oxford Box and AirSim manikin, respectively. A moderate correlation was found between collision count and global rating scale score. Overall these results suggest that collision count is a valid measure of fibreoptic scope handling skills. In addition, this study found a significant effect of gender on time taken to complete the Oxford Box task but not the manikin.

Other investigators have used tip surface collisions as a measure of fibreoptic scope handling skills [8-10]. There was, however, no discussion as to the validity of its use as an outcome measure. Our study suggests that collision count is a valid measure of fibreoptic handling skills and can be used in addition to other established outcome measures. Using tip collision count in training may improve handling skills of fibreoptic scope in manikins. Although our findings suggest that tip collision count is a useful measure of fibreoptic handling skills in manikins, there may be many reasons, such as secretions and blood, for loss of view in patients. However, using tip collisions in training may improve accuracy and precision of fibreoptic scope placement in clinical practice, which may lead to a reduction in the incidence of airway trauma. Further clinical studies are needed to establish this.

Only mild correlation was found between collision count and previous fibreoptic experience on the Oxford Box $(\rho=$ -0.24 ) and moderate correlation on the AirSim manikin ( $\rho=$ -0.42 ). There are a number of possible explanations for these findings. We relied on participants' recall of their previous fibreoptic experience. This could have led to an error in the reported numbers. Although a logbook record of fibreoptic experience may have been more accurate, this was unlikely to include manikin experience. Furthermore, lack of recent fibreoptic scope use may have influenced the correlation between collision count and previous fibreoptic experience. Forty out of 77 (52\%) volunteers had not used the fibreoptic scope in the preceding month.

This study found that gender has significant effect on time to task completion. Males completed the Oxford Box task faster, suggesting better visuospatial and psychomotor skills. This is consistent with what has been shown amongst surgeons and laparoscopic work [11]. Similarly, Williams et al. [13] found that males, on repeated attempts, were faster than females when performing a task on a novel fibreoptic trainer. However, we found no significant difference between genders in the AirSim manikin completion time. This could be due to task familiarity (in our institution the AirSim manikin is currently used for fibreoptic scope training) which may cancel out any inherent male advantage. Uneven and small group sizes may explain the absence of an effect of hand dominance and computer game use on fibreoptic scope handling skills, contrary to surgical data [11]. However, other investigators also failed to find a significant effect of hand dominance [13] or computer game experience [9] on performance. It may, therefore, be true that fibreoptic manipulation requires different visuospatial and psychomotor skills to laparoscopy.

Our study was conducted on two fibreoptic scope placement simulation tools. Using these simulation tools allowed for systematic evaluation of tip surface collisions in two constant test arrangements, eliminating variability present in real patient scenarios [14]. Furthermore, this allowed us to include volunteers with no, or limited, fibreoptic experience in order to determine more accurately the level of correlation. 
It may be regarded as unethical to allow volunteers with no fibreoptic experience to perform scope placement on patients. We therefore considered it unfeasible to carry out this study in the clinical setting.

In order to reduce variability in the collision counts, each video recording was viewed six times. This was time consuming and may be impractical in routine fibreoptic scope training. The previous patient study [7] that used collision count as an outcome measure did not describe its method of counting collisions from the video recording. Virtual reality models with tip collision counting facilities are available $[9,10]$. However, they are often costly when compared to the low fidelity simulators.

This study demonstrated strong and moderate correlations between collision count and task completion time. Using this outcome in addition to other measures of performance may improve accuracy and precision of fibreoptic scope placement and lead to improved safety of fibreoptic scope guided tracheal intubation. The results of this study infer that tip collision count can be used as a measure of fibreoptic performance in simulation based fibreoptic training, which may improve fibreoptic scope handling skills in clinical practice.

\section{Disclosure}

This study has been presented as a poster at the Euroanaesthesia 2010 meeting, Helsinki, Finland. Abstract publication: European Journal of Anaesthesiology 2010; 27: 260.

\section{Conflict of Interests}

The authors declare that there is no conflict of interests regarding the publication of this paper.

\section{Acknowledgments}

The authors would like to thank Pharmabotics Ltd. (Oxford Box) and TruCorp Ltd. (AirSim Bronchi) for donating equipment for the study and assisting the study.

\section{References}

[1] American Society of Anesthesiologists Task Force on Management of the Difficult Airway, "Practice guidelines for management of the difficult airway: an updated report by the American Society of Anesthesiologists Task Force on Management of the Difficult Airway," Anesthesiology, vol. 98, no. 5, pp. 1269-1277, 2003.

[2] J. J. Henderson, M. T. Popat, I. P. Latto, and A. C. Pearce, "Difficult Airway Society guidelines for management of the unanticipated difficult intubation," Anaesthesia, vol. 59, no. 7, pp. 675-694, 2004.

[3] T. Heidegger, H. J. Gerig, B. Ulrich, and T. W. Schnider, "Structure and process quality illustrated by fibreoptic intubation: analysis of 1612 cases," Anaesthesia, vol. 58, no. 8, pp. 734-739, 2003.
[4] V. N. Naik, E. D. Matsumoto, P. L. Houston et al., "Fiberoptic orotracheal intubation on anesthetized patients: do manipulation skills learned on a simple model transfer into the operating room?" Anesthesiology, vol. 95, no. 2, pp. 343-348, 2001.

[5] D. B. Chandra, G. L. Savoldelli, H. S. Joo, I. D. Weiss, and V. N. Naik, "Fiberoptic oral intubation: the effect of model fidelity on training for transfer to patient care," Anesthesiology, vol. 109, no. 6, pp. 1007-1013, 2008.

[6] K. Goldmann and T. Steinfeldt, "Acquisition of basic fiberoptic intubation skills with a virtual reality airway simulator," Journal of Clinical Anesthesia, vol. 18, no. 3, pp. 173-178, 2006.

[7] N. A. Crabtree, D. B. Chandra, I. D. Weiss, H. S. Joo, and V. N. Naik, "Fibreoptic airway training: correlation of simulator performance and clinical skill," Canadian Journal of Anesthesia, vol. 55, no. 2, pp. 100-104, 2008.

[8] R. Rowe and R. A. Cohen, "An evaluation of a virtual reality airway simulator," Anesthesia and Analgesia, vol. 95, no. 1, pp. 62-66, 2002.

[9] O. S. T. David, A. Derosiers, E. James Britt, A. M. Fein, M. L. Lesser, and A. C. Mehta, "Assessment of a bronchoscopy simulator," The American Journal of Respiratory and Critical Care Medicine, vol. 164, no. 12, pp. 2248-2255, 2002.

[10] H. G. Colt, S. W. Crawford, and O. Galbraith III, "Virtual reality bronchoscopy simulation: a revolution in procedural training," Chest, vol. 120, no. 4, pp. 1333-1339, 2001.

[11] T. P. Grantcharov, L. Bardram, P. Funch-Jensen, and J. Rosenberg, "Impact of hand dominance, gender, and experience with computer games on performance in virtual reality laparoscopy," Surgical Endoscopy and Other Interventional Techniques, vol. 17, no. 7, pp. 1082-1085, 2003.

[12] G. S. de Oliveira, R. Glassenberg, R. Chang, P. Fitzgerald, and R. J. McCarthy, "Virtual airway simulation to improve dexterity among novices performing fibreoptic intubation," Anaesthesia, vol. 68, no. 10, pp. 1053-1058, 2013.

[13] D. J. Williams, A. J. Byrne, and O. Bodger, "Validation of a novel fibreoptic intubation trainer," Anaesthesia, vol. 65, no. 1, pp. 1822, 2010.

[14] M. R. Rai and M. T. Popat, "Evaluation of airway equipment: man or manikin?” Anaesthesia, vol. 66, no. 1, pp. 1-3, 2011. 


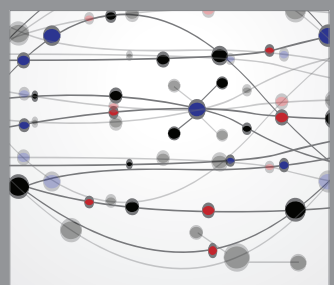

The Scientific World Journal
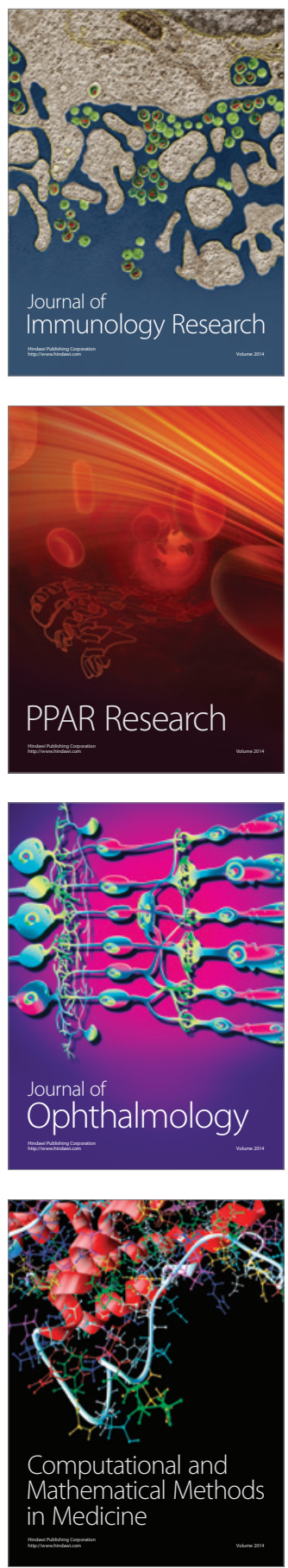

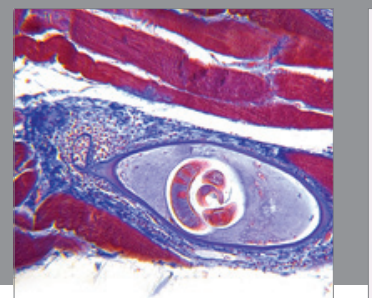

Gastroenterology

Research and Practice
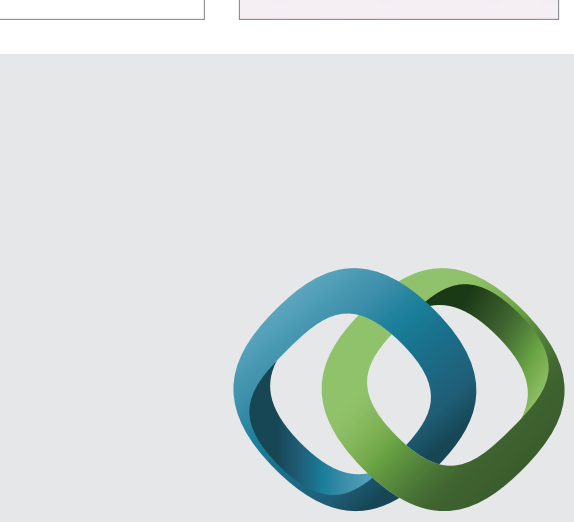

\section{Hindawi}

Submit your manuscripts at

http://www.hindawi.com
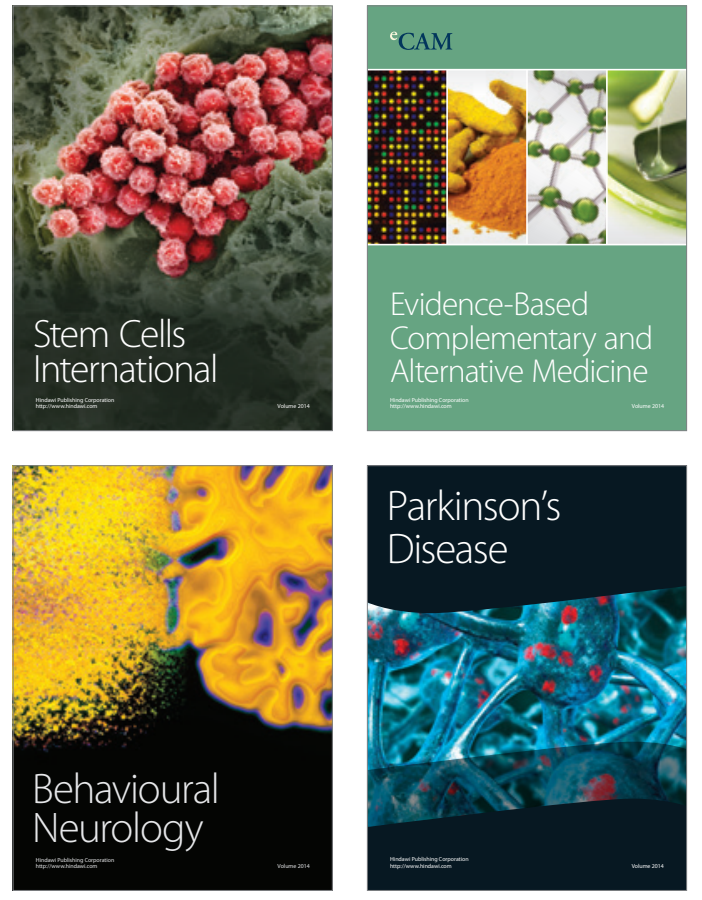
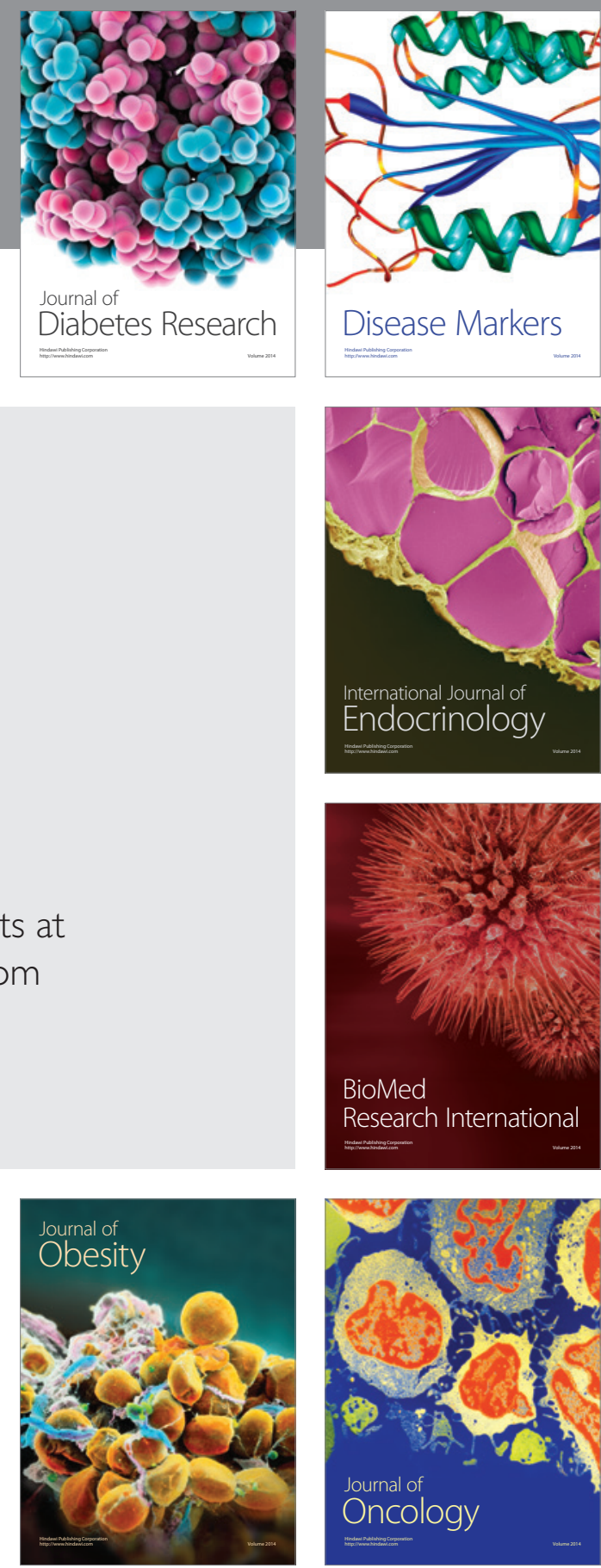

Disease Markers
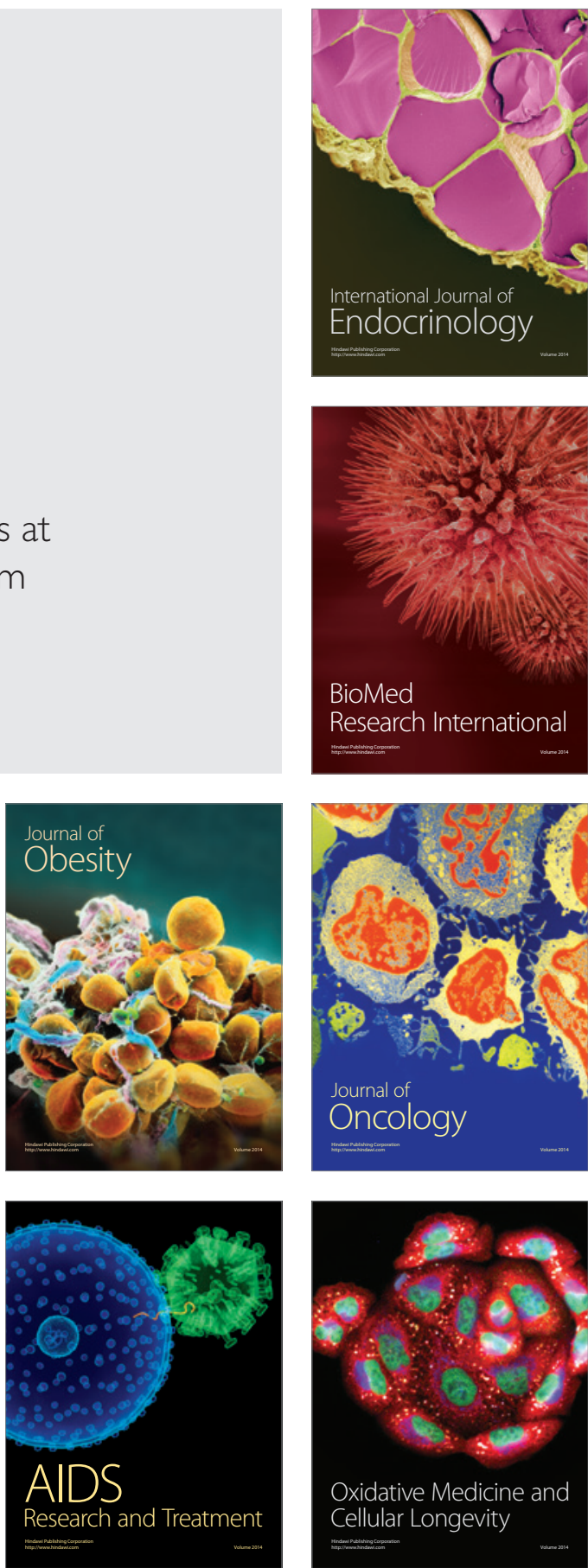